\title{
In vivo anti-nociceptive and anti-inflammatory activities of the aqueous extract of the leaves of Piper sarmentosum.
}

\begin{abstract}
Ethnopharmacological relevance: Piper sarmentosum (Piperaceae) is a medicinal plant traditionally used by the Malays to treat headaches, toothaches, coughs, asthma and fever. Aim of the study: In order to establish the pharmacological properties of the leaf of this plant, studies were performed on anti-nociceptive and anti-inflammatory activities. Materials and methods: The aqueous extract of Piper sarmentosum (AEPS) was prepared in the doses of 30, 100 and $300 \mathrm{mg} / \mathrm{kg}$. Anti-nociceptive activity of AEPS was evaluated by abdominal constriction and hot-plate tests. AEPS was also pre-challenged with $5 \mathrm{mg} / \mathrm{kg}$ naloxone to determine the involvement of opioid receptors. Anti-inflammatory activity was evaluated using carrageenan-induced paw edema assay. Results: Subcutaneous administration of AEPS exhibited anti-nociceptive activity $(\mathrm{P}<0.05)$ in a dose-dependent manner in the abdominal constriction and hot-plate tests. Pre-treatment with naloxone completely $(\mathrm{P}<0.05)$ diminished the extract anti-nociceptive activity in both tests. The AEPS, at all doses used, exerted significant $(\mathrm{P}<0.05)$ anti-inflammatory activity in a dose-dependent manner. Conclusions: The AEPS exhibits opioid-mediated anti-nociceptive activity at the peripheral and central levels, as well as anti-inflammatory activity, which confirmed the traditional uses of the plant in the treatment of pain- and inflammatory-related ailments.
\end{abstract}

Keyword: Anti-inflammatory; Anti-nociceptive; Aqueous extract; Opioids; Piper sarmentosum; Piperaceae. 